\title{
Impacto de un programa intracurricular sobre la disposición al estudio en universitarios
}

\author{
Fabiola M. Sáez-Delgado1, Alejandro E. Díaz-Mujica², Claudio E. Bustos ${ }^{3}$ y María V. Pérez-Villalobos ${ }^{2}$ \\ (1) Departamento Fundamentos de la Pedagogía, Facultad de Educación, Universidad Católica de la Santísima \\ Concepción, Chile (correo-e: fsaez@ucsc.cl) \\ (2) Departamento de Psicología, Facultad de Ciencias Sociales, Universidad de Concepción, Chile \\ (correo-e: adiazm@udec.cl; marperez@udec.cl) \\ (3) Departamento de Psiquiatría y Salud Mental, Facultad de Medicina, Universidad de Concepción, Chile \\ (correo-e: clbustos@udec.cl)
}

Recibido Nov. 15, 2019; Aceptado Ene. 10, 2020; Versión final Ene. 25, 2020, Publicado Ago. 2020

\begin{abstract}
Resumen
El objetivo de este trabajo fue evaluar el impacto de una intervención intracurricular en las estrategias y creencias de disposición al estudio en estudiantes de ingeniería. Se aplicó un programa de 12 sesiones de 20 minutos, aplicadas de forma intracurricular por los docentes de la asignatura Cálculo I. Se utilizó un diseño experimental aleatorizado por conglomerados con grupo control y experimental, con medición pre y post intervención. El tipo de muestreo fue no probabilístico en 5 carreras de ingeniería. Al azar, se asignaron 4 secciones con 117 estudiantes al grupo experimental y 3 secciones con 97 estudiantes al grupo control. Utilizando modelos lineales mixtos, se observaron mejores indicadores en las 9 variables dependientes del estudio en el grupo experimental. Se concluye que la intervención resulta efectiva para potenciar la disposición al estudio en alumnos de ingeniería.
\end{abstract}

Palabras clave: autorregulación del aprendizaje; disposición al estudio; entrenamiento; enseñanza-aprendizaje

\section{Impact of an intra-curricular program on disposition to study among university students}

\begin{abstract}
The main objective of this research study was to evaluate the impact of an intra-curricular intervention on the strategies and beliefs of disposition to study in engineering students. A program of 12 sessions of 20 minutes was applied by professors teaching the subject Calculus I. An experimental design randomized by conglomerates with a control and an experimental group was used, with pre and post intervention measurements. The type of sampling was no-probabilistic in five engineering careers. Four sections with 117 students were assigned randomly to the experimental group and three sections with 97 students were assigned to the control group. Using mixed linear models, the results showed better indicators in the experimental group for the nine dependent variables in the study. It is concluded that the intervention is effective to enhance the disposition to study in engineering students.
\end{abstract}

Keywords: self-regulated learning; disposition to study; training; teaching-learning 


\section{INTRODUCCIÓN}

El fracaso académico y abandono precoz es una preocupación de la sociedad actual y particularmente de la comunidad educativa, por la dimensión del fenómeno y consecuencias sociales (Lattuada, 2017). De hecho, el impacto social y económico que ha implicado la deserción estudiantil universitaria, lo ha transformado en un aspecto prioritario en la Educación Superior (Burke, 2019). En general, las comunidades universitarias esperan que sus estudiantes lleguen desde la educación secundaria altamente capacitados para afrontar sus desafíos académicos con éxito, y con la claridad de que este depende principalmente de su esfuerzo. Sin embargo, la transición del nivel educativo anterior a la universidad implica diversos desafíos en el proceso de adaptarse a la vida universitaria, incluida una nueva estructura institucional y el requerimiento de un perfil protagonista del proceso de aprendizaje por parte del estudiante, lo que acrecienta y destaca la importancia de la capacidad de autorregulación a la hora de afrontar los estudios, proceso que no es promovido por la universidad. Específicamente, se ha identificado que en los primeros años de universidad que los estudiantes presentan un insuficiente desarrollo de estrategias y creencias de disposición al estudio (King et al., 2019).

La disposición al estudio se define como las habilidades cognitivo-motivacionales en los procedimientos iniciales del estudio y aprendizaje y corresponde a la primera etapa del proceso de autorregulación del aprendizaje (ARA) (Sáez et al., 2018b). La etapa de disposición al estudio implica desplegar un junto de estrategias y creencias que permiten una actitud, motivación, estado emocional y planificación estratégica con el objetivo de alcanzar exitosamente las tareas académicas y el logro del aprendizaje (Dowd et al., 2019). Respecto de las creencias de disposición al estudio son dos y corresponden a creencias de autoeficacia y atribucionales. Las atribuciones causales son comprendidas como un conjunto de creencias que se perciben por parte del estudiante como responsables ante un determinado resultado académico, las que permiten informar las representaciones que los estudiantes tienen respecto del esfuerzo puesto y del resultado alcanzado en su desempeño en la tarea (Pérez-Villalobos et al., 2018), estableciendo una relación causal entre ambos (Weiner, 2010). La autoeficacia para la autorregulación de la disposición al estudio es definida como las creencias sobre la propia capacidad que tiene un estudiante para el logro de la autorregulación del uso de estrategias conducentes a preparar la actividad de estudio (Sáez et al., 2018a).

Respecto de las estrategias de disposición al estudio son conductas planificadas que tienen por objetivo iniciar de manera estratégica la actividad de estudio. Las principales estrategias de disposición al estudio son tres: (a) establecimiento de objetivos de tipo académicos, (b) gestión del tiempo académico y (c) organización de recursos materiales y del ambiente. El establecimiento de objetivos académicos se refiere a la claridad de un propósito de comportamiento para el logro de un determinado aprendizaje dentro de un período de tiempo establecido (Bloom, 2013). La gestión del tiempo académico se refiere al conocimiento y uso de los espacios temporales necesarios para desarrollar una tarea, y para el logro de los resultados de aprendizaje (Wang et al., 2019). Por último, la organización de recursos materiales y ambientales se refiere al ordenamiento de los recursos correspondientes a los materiales necesarios para realizar una tarea de aprendizaje y el ordenamiento de los recursos del ambiente para la adaptación de las condiciones del lugar con el que se cuenta para realizar la tarea de aprendizaje (García-Ros y Pérez-González, 2011).

Las formas de promoción de la autorregulación del aprendizaje en contextos universitarios se clasifican en programa en formato extracurricular e intracurricular (Sáez et al., 2018c). Los programas extracurriculares consisten en cursos de preparación llamados "aprender a aprender", intervenciones específicas, cursos de nivelación desarrollados en el verano o programas puente entre la secundaria y la universidad, conducentes a cubrir lagunas, focalizados en mejorar estrategias aisladas. Los programas que promueven la autorregulación del aprendizaje de tipo intracurricular se caracterizan por facilitar en el aula por los propios docentes el empleo de estrategias y creencias autorregulatorias de disposición al estudio, siendo promovidas y enseñadas en conjunto con los contenidos curriculares correspondiente a cada asignatura (Díaz et al., 2017).

Si bien se han diseñado programas de intervención para promover procesos de autorregulación, estudios publicados sobre programas intracurriculares son escasos, con evidencia empírica de resultados, y los mismos autores describen importantes limitaciones en su desarrollo y aplicación (Sáez et al., 2018c). Se considera que esta modalidad de intervenciones está en un estado inicial de desarrollo. Por lo tanto, es conveniente desarrollar programas intracurriculares para el fomento de estrategias y creencias de disposición al estudio en estudiantes universitarios, y evaluar su efectividad (Hernández y Camargo, 2017; Zeidner, 2019).

El objetivo de esta investigación fue evaluar el impacto de una intervención intracurricular para la disposición al estudio en estudiantes universitarios. Se plantearon 3 hipótesis: (1) La exposición al Programa Intracurricular de Disposición al estudio aumenta las puntuaciones en las estrategias establecimiento de objetivos académicos, gestión del tiempo académico, y organización de recursos materiales y ambientales; (2) La exposición al Programa Intracurricular de Disposición al estudio mejora las creencias de autoeficacia para la autorregulación de la disposición al estudio; y (3) la exposición al Programa Intracurricular de Disposición al estudio mejora las creencias de atribuciones causales. 


\section{MÉTODO}

Se utilizó un diseño experimental por conglomerados, ya que se asignaron de forma aleatoria cursos completos al grupo experimental al grupo control, con medición de pre y postest. En término de las variables involucradas, corresponde a un diseño mixto $2 X 2$ con un factor intra sujeto (el tiempo), y un factor intersujeto (control o experimental).

\section{Participantes}

La intervención tuvo una participación total de 214 estudiantes de ingeniería de la Universidad Católica de la Santísima Concepción, de cinco carreras. El grupo experimental (G.E) estuvo compuesto por 4 secciones, correspondientes a las carreras de Ingeniería civil, ingeniería geológica, ingeniería informática e ingeniería industrial. Los 117 estudiantes del grupo experimental se dividían en 34 mujeres (29,05\%) y 83 hombres (70,94\%), con una media de edad de 18,66 años de edad ( $D E=1,28)$. El grupo control (G.C) estuvo compuesto por 3 secciones, dos de ingeniería industrial y una de ingeniería electrónica. E grupo de 97 alumnos del grupo control estaba conformado por 27 mujeres $(27,83 \%)$ y 70 hombres $(72,16 \%)$, con una media de edad de 18,57 años $(\mathrm{DE}=1,13)$.

\section{Programa de intervención}

El objetivo general del Programa fue desarrollar estrategias y creencias autorregulatorias de disposición al estudio en estudiantes que cursan primer año de universidad en carreras de Ingeniería con el fin de mejorar su disposición para aprender. Implicó 14 clases, la primera clase es para la presentación del programa, firma de consentimientos informados y aplicación de instrumentos. La última clase es para el cierre del programa y aplicación de instrumentos. Las 12 clases (Tabla 1) destinadas a la ejecución del Programa realizadas con una frecuencia de una por semana corresponden a 4 horas en el aula (20 minutos cada clase), y 4 horas de trabajo autónomo (20 minutos de tareas por sesión). La modalidad de aplicación fue de tipo Intracurricular en la segunda unidad de la asignatura Cálculo I. Estaba dirigido a estudiantes que cursan por primera vez primer año de la carrera de Ingeniería. Todas las actividades desarrolladas por el docente para cumplir con los resultados de aprendizaje de la asignatura implicaban también los resultados de aprendizaje del programa. Los ejemplos y situaciones de aplicación de las estrategias y creencias fueron entregados a los estudiantes a través de dos maneras: (1) mediante instrucciones verbales del docente de la asignatura, y (2) mediante un texto tanto de contenidos como de actividades.

Tabla 1: Nombre de cada sesión y resultados de aprendizaje del Programa.

\begin{tabular}{|c|c|}
\hline Nombre de cada sesión & Resultado de aprendizaje \\
\hline $\begin{array}{l}\text { Sesión } 1 . \\
\text { Mis dificultades frente al estudio }\end{array}$ & $\begin{array}{l}\text { Identifica dificultades en el uso de } 3 \text { estrategias de disposición al estudio: (1) } \\
\text { establecimiento de objetivos académicos, (2) gestión del tiempo académico, y } \\
\text { (3) organización de recursos materiales y ambientales. }\end{array}$ \\
\hline $\begin{array}{l}\text { Sesión } 2 . \\
\text { Mejorando mi estudio }\end{array}$ & $\begin{array}{l}\text { Reconoce } 3 \text { estrategias de disposición al estudio. } \\
\text { Propone un cambio de mejora en el comportamiento de estudio para cada } \\
\text { estrategia. }\end{array}$ \\
\hline $\begin{array}{l}\text { Sesión } 3 . \\
\text { Establezco objetivos }\end{array}$ & $\begin{array}{l}\text { Redacta } 3 \text { objetivos académicos para la asignatura de Cálculo I, considerando } \\
\text { las propiedades de los objetivos (concretos, realistas, evaluables). }\end{array}$ \\
\hline $\begin{array}{l}\text { Sesión } 4 . \\
\text { Plazo para mis objetivos }\end{array}$ & $\begin{array}{l}\text { Redacta } 3 \text { objetivos académicos para la asignatura de Cálculo I, considerando } \\
\text { la temporalidad (corto, mediano y largo plazo). }\end{array}$ \\
\hline $\begin{array}{l}\text { Sesión } 5 . \\
\text { Planificando la semana y las } \\
\text { actividades fijas }\end{array}$ & $\begin{array}{l}\text { Diseña planificación semanal. } \\
\text { Anota actividades fijas. }\end{array}$ \\
\hline $\begin{array}{l}\text { Sesión } 6 . \\
\text { Mis prioridades }\end{array}$ & Diseña una lista de cosas por hacer e indica cuál de ellas es prioridad. \\
\hline $\begin{array}{l}\text { Sesión } 7 . \\
\text { Reflexiono de forma constructiva sobre } \\
\text { las causas de mis fracasos en mi } \\
\text { desempeño }\end{array}$ & $\begin{array}{l}\text { Autoevalúa las causas de un fracaso en su desempeño en la asignatura de } \\
\text { Cálculo I: } \\
\text { Se centra en errores de procedimiento empleado. } \\
\text { Descarta la falta de capacidad, la suerte y factores externos a él mismo. }\end{array}$ \\
\hline $\begin{array}{l}\text { Sesión } 8 . \\
\text { Reflexiono de forma constructiva sobre } \\
\text { las causas de mis éxitos en mi } \\
\text { desempeño }\end{array}$ & $\begin{array}{l}\text { Autoevalúa las causas de desempeño exitoso en la asignatura de Cálculo I: } \\
\text { Valora su esfuerzo y su método. } \\
\text { Valora su capacidad y sus destrezas. } \\
\text { Descarta la suerte y diversos factores a él mismo. }\end{array}$ \\
\hline
\end{tabular}


Tabla 1: (continuación)

\begin{tabular}{|l|l|}
\hline $\begin{array}{l}\text { Sesión 9. } \\
\text { Tengo todo lo necesario para estudiar }\end{array}$ & - Organiza los materiales necesarios para un estudio eficaz. \\
\hline $\begin{array}{l}\text { Sesión 10. } \\
\text { Mejoro mi lugar de estudio }\end{array}$ & - Busca un lugar óptimo para estudiar. \\
\hline $\begin{array}{l}\text { Sesión } 11 . \\
\begin{array}{l}\text { Reflexiono sobre mi autoeficacia para } \\
\text { el estudio }\end{array}\end{array}$ & $\begin{array}{l}\text { - Autoevalúa su eficacia para: (a) establecer objetivos } \\
\text { académicos, (b) gestionar el tiempo académico y (c) organizar los recursos de } \\
\text { tipo material y los referidos al ambiente de estudio. }\end{array}$ \\
\hline $\begin{array}{l}\text { Sesión 12. } \\
\text { Empleando estrategias de disposición } \\
\text { al estudio }\end{array}$ & $\begin{array}{l}\text { - Diseña un plan para preparar el tercer certamen de Cálculo I utilizando } \\
\text { estrategias autorregulatorias de disposición al estudio: (a) establece } \\
\text { objetivos académicos, (b) gestiona su tiempo de estudio y (c) organiza sus } \\
\text { materiales y lugar de estudio. }\end{array}$ \\
\hline
\end{tabular}

\section{Instrumento}

Se construyó un cuestionario a partir de otros instrumentos disponibles previamente (García-Ros y PérezGonzález, 2012; Inglés et al., 2008; Sáez et al., 2018a), y fueron validadas siguiendo las directrices de diseño, construcción y adaptación de escalas de estrategias de estudio y autorregulación. El cuestionario estaba compuesto de 9 subdimensiones (ver Tabla 2). En todas las subdimensiones se respondió en escala Likert de 1 a 5 puntos.

Tabla 2: Consistencia interna de las subescalas del Cuestionario de Disposición al Estudio.

\begin{tabular}{|l|c|c|c|}
\hline Escala & Abreviación & $\begin{array}{c}\text { Alpha de } \\
\text { Cronbach }(\alpha)\end{array}$ & $\begin{array}{c}\text { Numero } \\
\text { de ítems }\end{array}$ \\
\hline 1. Estrategias de disposición al estudio & & & \\
\hline Establecimiento de objetivos académicos & $(\mathrm{EOA})$ & .82 & 8 \\
\hline Gestión del tiempo académico & $(\mathrm{GTA})$ & .82 & 4 \\
\hline Organización de recursos materiales y ambientales & (ORMA) & .70 & 4 \\
\hline 2. Atribuciones causales en la autorregulación de la disposición al estudio & & & \\
\hline De éxito al esfuerzo & (ACEE) & .84 & 5 \\
\hline De éxito a la habilidad & (ACEH) & .81 & 5 \\
\hline De fracaso al esfuerzo & (ACFE) & .82 & 5 \\
\hline De fracaso a la habilidad & (ACFH) & .78 & 5 \\
\hline De fracaso a factores externos & (ACFE) & .59 & 3 \\
\hline 3. Autoeficacia para la autorregulación de la disposición al estudio & (AUTOEF) & .84 & 9 \\
\hline
\end{tabular}

\section{Procedimiento}

Se realizó una validación del programa de intervención por jueces expertos y aplicación piloto. Luego se solicitaron las autorizaciones a autoridades de la universidad y los docentes de los cursos específicos para aplicar el programa en la asignatura de Cálculo I, por sus altas tasas de reprobación y por ser común en el plan de estudios de las diferentes carreras de ingeniería. Para la asignación de los cursos a los grupos experimental y control, se realizó un sorteo al azar entre los docentes que aceptaron participar del estudio. Así, los docentes que sortearon ser parte del grupo experimental aplicarían el Programa en el desarrollo de su clase. Los criterios de inclusión de la muestra de estudiantes de los grupos control y experimental fueron ser estudiante que cursaban por primera vez la asignatura de cálculo I.

El primer semestre del año académico se planificó la aplicación de las 12 sesiones del Programa. Previo al inicio de las sesiones, se aplicó el pre-test, momento en el cual se explicó a los estudiantes el objetivo, metodología de la investigación, actividades a desarrollar, y se les invitó a participar. Aquellos que aceptaron y firmaron el consentimiento informado, se les aplicó los instrumentos de medida. Los docentes se reunieron en el horario coordinado con la investigadora responsable de esta investigación para precisar procedimientos y protocolos previamente a cada aplicación de las sesiones del Programa. Al finalizar las 12 sesiones, en una clase posterior, se realizó la aplicación del post-test. Este estudio fue aprobado por el Comité de ética del Departamento de Psicología de la Universidad de Concepción, Chile. 


\section{Análisis de datos}

Para analizar el efecto de la intervención, se utilizaron modelos lineales mixtos. Se consideró como efectos fijos el momento de aplicación (pre-post), el grupo del tratamiento (experimental, control) y también el efecto de interacción entre el momento de aplicación y el grupo de tratamiento, que daría cuenta de la existencia de un efecto de la intervención. Como efectos aleatorios se utilizó un modelo de solo intercepto para los individuos y los cursos. Para estimar el tamaño del efecto de la intervención, se utilizó el eta generalizado ( $\left.\eta_{G}\right)$ para modelos mixtos (Olejnik y Algina, 2003) en términos de modelos generales, y tamaño del efecto para el impacto particular de la intervención por grupo experimental y control.

\section{RESULTADOS}

En la tabla 3 (ver notación para interpretación de símbolos) se observan los resultados obtenidos de la prueba de interacción entre el tiempo y el tratamiento, y los contrastes para las variables de disposición al estudio. Se puede observar que existe un efecto de interacción significativo tanto en las variables asociadas a las estrategias de disposición al estudio como a las de atribuciones causales de éxito y fracaso, como también para la autoeficacia. En todos los casos se observa que el grupo experimental presenta un aumento mayor al grupo control en el mismo período para todas las variables de estudio, excepción de las Atribuciones causales de fracaso a la habilidad y factores externos, que disminuyen. Esto es deseable en el grupo experimental, debido a que se espera que los estudiantes mejoren la calidad de la atribución. Es decir, que los estudiantes hagan atribuciones de fracaso a su esfuerzo y no a su habilidad o factores externos.

Con respecto al grupo control se observa que las diferencias (Dif.) para la mayoría de las variables disminuyen, no resultando significativas además para la gestión del tiempo y las atribuciones causales de éxito y fracaso al esfuerzo y la habilidad. Respecto del grupo experimental se observa un mejora estadísticamente significativo en todas las variables con un efecto pequeño entre 0.01 y 0.05 , a excepción de la variable establecimiento de objetivos académicos que se encontró un efecto medio de 0.1 (Olejnik y Algina, 2003).

Tabla 3: Contraste de efecto de intervención para las estrategias y creencias de disposición al estudio (Modelo 1).

\begin{tabular}{|c|c|c|c|c|c|}
\hline Modelo 1 & $x^{2}(1)$ & $\begin{array}{c}\text { Dif. de medias del } \\
\text { G.C entre el } \\
\text { post y pre test }\end{array}$ & $\begin{array}{c}\text { Dif. de medias del } \\
\text { G.E entre el } \\
\text { post y pre test }\end{array}$ & $\begin{array}{l}\text { Dif. en dif. entre la media del G.E } \\
\text { entre el post y pre test y la media } \\
\text { del G.C entre el post y pre test }\end{array}$ & $\eta_{G}$ \\
\hline \multicolumn{6}{|c|}{ Estrategias de disposición al estudio } \\
\hline EOA & $28.09^{* *}$ & $-0.25^{\star *}$ & $1.09^{* *}$ & $1.34^{\star *}$ & 0.10 \\
\hline GTA & $17.44^{* *}$ & -0.14 & $1.38^{* *}$ & $1.52^{* *}$ & 0.03 \\
\hline ORMA & $20.05^{\star *}$ & $-0.41^{* *}$ & $0.96^{\star *}$ & $1.37^{\star \star}$ & 0.04 \\
\hline \multicolumn{6}{|c|}{ Atribuciones causales de éxito } \\
\hline Esfuerzo & $7.33^{* *}$ & 0.02 & $0.61^{\star *}$ & $0.59^{* *}$ & 0.01 \\
\hline Habilidad & $9.40^{* *}$ & -0.00 & $0.44^{\star *}$ & $0.44^{\star \star}$ & 0.01 \\
\hline \multicolumn{6}{|c|}{ Atribuciones causales de fracaso } \\
\hline Esfuerzo & $12.61^{* *}$ & -0.14 & $0.30^{\star *}$ & $0.44^{* *}$ & 0.01 \\
\hline Habilidad & $11.13^{* *}$ & 0.19 & $-0.39^{\star *}$ & $-0.58^{* *}$ & 0.01 \\
\hline Causas Externas & $18.69^{* *}$ & $0.22^{\star *}$ & $-0.64^{* *}$ & $-0.86^{* *}$ & 0.05 \\
\hline AUTFC & $22.13^{* *}$ & $-0.38^{* *}$ & 1.00 ** & $1.38^{\star *}$ & 0.05 \\
\hline
\end{tabular}

Al analizar los efectos principales de tiempo y grupo además de la interacción entre ambos; se puede observar que el efecto de interacción es significativo y positivo en todas las variables, ratificando la existencia de un efecto de la intervención en todas las escalas medidas. Con respecto a los efectos aleatorios la mayor fuente de variabilidad se observó a nivel de sujeto, seguido por el residuo que surge a nivel aplicación y finalmente la diferencia entre secciones. Esto quiere decir que las diferencias individuales permanecen a lo largo del desarrollo del programa, siendo mayores a las que se observan entre los grupos. El análisis de residuos mostró que los modelos son lineales ya que los residuos están cercanos a cero para cada media de la 
interacción grupo momento. Los residuos a nivel de aplicación son normales en todas las variables, así como los efectos aleatorios a nivel de individuos y de tiempo inicial de secciones, pero se observó ausencia de normalidad en los residuos a nivel de secciones en el momento 2; en particular, se notó que ingeniería civil presentó un aumento mayor en los puntajes del post respecto de las otras secciones. Por tanto, se consideró un segundo modelo donde se tuvo en cuenta el efecto de la intervención específico en ingeniería civil.

La tabla 4 muestra el modelo 2 (ver notación para interpretación de símbolos) que considera el efecto diferencial de pertenecer o no a la carrera de ingeniería civil en los puntajes de las diferentes variables. Usando la prueba de razón de verosimilitud, se probó en cada variable si existía un efecto de interacción grupo $\mathrm{X}$ tiempo distinto para ingeniería civil que para el resto de las secciones. En general, los tamaños del efecto en estas variables son mayores en la sección ingeniería civil en comparación con los tamaños del efecto de las otras secciones. Se observa que este fenómeno aplica para las variables de estrategias de disposición (establecimiento de objetivos académicos, gestión del tiempo académico y organización de recursos materiales y ambientales), autoeficacia para la autorregulación de la disposición al estudio y en atribuciones causales de éxito al esfuerzo. Al considerar sólo la sección ingeniería civil se observa un efecto grande en las cinco variables (entre $\eta=0.30$ y $\eta=1.08$ ); en el resto de las secciones, se ve un tamaño del efecto entre pequeño $(\eta=0,01)$ y medio $(\eta=0,11)$. Al analizar los efectos aleatorios podemos observar que se mantienen similares las varianzas a nivel de individuos y de residuo en la aplicación, pero disminuye la varianza a nivel de secciones; esto nos indica que en el primer modelo las diferencias de ingeniería civil con el resto de las secciones explica esta varianza.

Tabla 4: Contraste de efecto de intervención para las estrategias y creencias de disposición al estudio (Modelo 2).

\begin{tabular}{|c|c|c|c|c|c|c|c|}
\hline \multirow[t]{2}{*}{ Modelo 2} & \multicolumn{5}{|c|}{ Todas las secciones menos Ingeniería civil } & \multicolumn{2}{|c|}{ Sólo en Ing. Civil } \\
\hline & $X^{2}(2)$ & $\begin{array}{l}\text { Dif. de medias } \\
\text { del G.C entre } \\
\text { el post y pre } \\
\text { test de todas } \\
\text { las secciones } \\
\text { menos Ing. } \\
\text { Civil }\end{array}$ & $\begin{array}{l}\text { Dif. de medias } \\
\text { del G.E entre } \\
\text { el post y pre } \\
\text { test de todas } \\
\text { las secciones } \\
\text { menos Ing. } \\
\text { Civil }\end{array}$ & $\begin{array}{l}\text { Dif. en dif.entre la } \\
\text { media del G.E entre } \\
\text { el post y pre test y la } \\
\text { media del G.C entre } \\
\text { el post y pre test de } \\
\text { todas las secciones } \\
\text { menos Ing. Civil }\end{array}$ & $\eta_{G}$ & $\begin{array}{c}\text { Dif. de } \\
\text { medias del } \\
\text { G.E entre el } \\
\text { post y pre } \\
\text { test sólo en la } \\
\text { sección Ing. } \\
\text { Civil }\end{array}$ & $\eta G$ \\
\hline \multicolumn{8}{|c|}{ Estrategias de disposición al estudio } \\
\hline EOA & $6.38^{*}$ & $-0.25^{* *}$ & $1.02^{\star *}$ & $1.27^{\star *}$ & 0.11 & $1.32^{* *}$ & 0.38 \\
\hline GT & $7.54^{\star}$ & -0.14 & $1.23^{\star *}$ & $1.38^{* *}$ & 0.04 & $1.82^{* *}$ & 1.08 \\
\hline ORMA & $14.49^{* *}$ & -0.41 & $0.81^{\star *}$ & $1.21^{\star *}$ & 0.10 & $1.42^{* *}$ & 0.38 \\
\hline \multicolumn{8}{|c|}{ Atribuciones causales de éxito } \\
\hline esfuerzo & $9.99^{* *}$ & 0.01 & $0.45^{\star *}$ & $0.44^{\star *}$ & 0.01 & $1.09^{* *}$ & 0.30 \\
\hline Habilidad & 0.95 & 0 & $0.48^{\star *}$ & $0.48^{* *}$ & 0.01 & $0.33^{* *}$ & 0.03 \\
\hline \multicolumn{8}{|c|}{ Atribuciones causales de fracaso } \\
\hline Esfuerzo & 1.18 & -0.14 & $0.27^{\star \star}$ & $0.41^{\star \star}$ & 0.01 & $0.39^{* *}$ & 0.04 \\
\hline Habilidad & 1.58 & 0.18 & $-0.42^{* *}$ & $-0.61^{* *}$ & 0.01 & $-0.29^{* *}$ & 0.04 \\
\hline $\begin{array}{l}\text { Factores } \\
\text { externos }\end{array}$ & 5.67 & 0.22 * & $-0.71^{* *}$ & $-0.93^{\star *}$ & 0.05 & $-0.43^{* *}$ & 0.10 \\
\hline AUTFC & $11.39^{* *}$ & $-0.38^{* *}$ & $0.88^{* *}$ & $1.26^{\star *}$ & 0.10 & $1.36^{* *}$ & 0.45 \\
\hline
\end{tabular}

\section{DISCUSIÓN}

El objetivo del presente estudio fue evaluar el impacto de una intervención intracurricular para la disposición al estudio en estudiantes universitarios. El principal hallazgo es que el Programa de entrenamiento para la disposición al estudio fue efectivo en el desarrollo y mejora de competencias autorregulatorias de disposición al estudio. Se debe destacar que se observó un efecto diferencial por carrera, siendo los tamaños del efecto para la sección de Ingeniería Civil mayores a los del resto de las carreras. La claridad de los resultados de la efectividad de los programas es un antecedente determinante a la hora de desarrollar una intervención, escogiendo un programa que evidencie empíricamente resultados de mejora para implementar este en una determinada realidad educativa. Sin embargo, no se suele reportar información estadística que permita observar estimadores de efectividad. Los resultados de este estudio aportan evidencia teórica y empírica a los escasos estudios que muestran el impacto de programas promotores de procesos de autorregulación en Iberoamérica (Hernández y Camargo, 2017). 


\section{Respecto de la comprobación de las hipótesis del estudio}

En relación con la hipótesis 1: "la exposición al Programa Intracurricular de Disposición al estudio aumenta las puntuaciones en las estrategias establecimiento de objetivos académicos, gestión del tiempo académico, y organización de recursos materiales y ambientales", el efecto de la intervención sobre las estrategias de disposición al estudio en la sección de ingeniería civil fue de tamaño grande, mientras que en el resto de las secciones el tamaño del efecto fue entre pequeño y medio. En relación con la hipótesis 2 "La exposición al Programa Intracurricular de Disposición al estudio mejora las creencias de autoeficacia para la autorregulación de la disposición al estudio", se observa que el efecto de la intervención sobre las creencias de autoeficacia para la autorregulación de la disposición al estudio en la sección de ingeniería civil fue grande, mientras que para el resto de las secciones fue medio. Finalmente, para la hipótesis 3 "la exposición al Programa Intracurricular de Disposición al estudio mejora las creencias de atribuciones causales", se puede apreciar que el efecto de la intervención sobre las atribuciones causales de éxito al esfuerzo en la sección ingeniería civil fue grande, mientras que para el resto de las secciones el tamaño del efecto de la intervención sobre las atribuciones causales de éxito al esfuerzo fue pequeño.

\section{Principales factores que podrían explicar la efectividad del programa}

La efectividad del Programa intrauricular desarrollado sobre las estrategias y creencias para la disposición al estudio en universitarios podría explicarse por al menos tres motivos: (1) fundamentado en la teoría, (2) estructura de las sesiones del Programa (3) modalidad de aplicación en formato intracurricular.

En primer lugar, el programa se fundamenta en la convergencia teoría de los modelos más importantes de autorregulación del aprendizaje. Esto permitió la coherencia lograda entre las actividades, objetivos y resultados de aprendizaje del Programa, en base a desarrollar las estrategias y creencias de disposición al estudio, y en la pertinencia del modelo teórico de autorregulación del aprendizaje como proceso cíclico de tres fases: disposición, desempeño y evaluación (Zeidner, 2019).

La segunda razón que destacamos es el poder contar con una guía que estructuró las sesiones (en forma de manual de trabajo) para los estudiantes, ayudó a centrarse en temáticas dirigidas y concretas de conocimiento, lo que permitió el logro de la característica de un programa intracurricular (Loeffler et al., 2019). Así mismo, se realizó un acompañamiento individual a los docentes participantes, el que consistió en una reunión semanal coordinada según la disponibilidad del profesor y antes de la aplicación de cada sesión de la intervención realizada a sus estudiantes, sumado a retroalimentaciones posteriores a cada sesión, esto podría haber ayudado a que las estrategias y creencias se fomentaran de manera más consciente y clara, lo que podría haber impactado en la implicación de los profesores para facilitar la promoción de competencias autorregulatorias y consecuentemente en la efectividad de la intervención (Barr y Askell-Williams, 2019).

En tercer lugar, probablemente parte del efecto de este programa se debe precisamente a su característica intracurricular. De acuerdo a la literatura, una estrategia de instrucción docente para la promoción de variables de autorregulación del aprendizaje incluidas en el plan de estudios de una asignatura aumenta la probabilidad de que los estudiantes apliquen las competencias autorregulatorias a los contenidos que están aprendiendo en la misma asignatura (Duchatelet y Donche, 2019). Las condiciones de esta característica es la implicación del docente de asignatura disciplinar en el desarrollo de competencias de autorregulación, y el desarrollo de actividades específicas en el contexto de la asignatura. Investigaciones recientes coinciden con estas afirmaciones, y establecen que las condiciones del entrenamiento aplicado son centrales para el diseño de intervenciones orientadas a mejorar la autorregulación del aprendizaje: (1) seleccionar un conjunto específico de procedimientos, (2) observar evidencias de sus resultados, (3) situarlo en el contexto del aula, de acuerdo con los objetivos y características propias de una determinada asignatura, (4) ser facilitado por el docente de la asignatura (Díaz et al., 2017).

\section{Elección de participantes y efecto diferencial}

La elección de los participantes se basó en la revisión de la literatura. En carreras de ingeniería se presenta los fenómenos de fracaso académico y deserción, especialmente durante los primeros semestres del plan de estudio (Pinxten et al., 2019). Uno de los factores que explica el fracaso académico es la falta de autorregulación del aprendizaje (independientemente del nivel intelectual) (Graffigna et al., 2014). Resultados de estudios en carreras de ingeniería, han mostrado deficiencias en el modo en que los estudiantes aprenden las distintas asignaturas, específicamente procesos de autorregulación (Lawanto, Febrian, Butler, \& Mina, 2019). El $80 \%$ de estudiantes de ingeniería tiene deficiencias serias en el uso de estrategias de autorregulación, lo que se traduce en bajo rendimiento académico. Los alumnos ingresan a la universidad sin hábitos de estudio autorregulados (Concannon et al., 2019). 
A pesar de la importancia de la promoción de competencias de autorregulación en estudiantes de ingeniería, su desarrollo está en niveles iniciales (Ali, 2019). Se han realizado mejoras en la educación en ingeniería, produciendo avances centradas en el estudiante, sin embargo, no ha habido un cambio sistémico importante. Por lo tanto, fomentar innovaciones requiere atención adicional (Concannon et al., 2019; Pinxten et al., 2019). La presente investigación asumió el reto de implementar al interior de los planes de estudio de carreras de ingeniería un programa de intervención con énfasis en el desarrollo y uso de estrategias y creencias de autorregulación del aprendizaje en el primer semestre de carreras de ingeniería (Lawanto et al., 2019).

Los resultados mostraron un tamaño del efecto distinto del Programa en los participantes dependiendo si estos pertenecían a la carrera de ingeniería civil o eran estudiantes de otra ingeniería (industrial, geológica e informática). Es un aporte mostrar el efecto diferenciador del impacto del Programa considerando la pertenencia de los estudiantes a una determinada carrera, puesto que los resultados de los estudios que buscan mejorar variables de autorregulación del aprendizaje en estudiantes universitarios no muestran estas especificaciones aun cuando en su estudio participan estudiantes de diferentes carreras. Parece justificable suponer que, de acuerdo de las características de los grupos participantes en Programas de intervención, algunos grupos podrían beneficiarse más del entrenamiento. Con respecto al diseño de programas de entrenamiento de ARA en la universidad, es necesario saber que los diferentes grupos de estudiantes no se benefician en la misma medida de una intervención ARA (de la Fuente et al., 2018).

Es de gran importancia examinar si un determinado Programa de entrenamiento es efectivo sólo para grupos específicos de estudiantes o si puede usarse de manera ubicua. Además, es necesario investigar los efectos del tratamiento considerando la heterogeneidad del grupo porque en caso contrario se pueden hacer interpretaciones erróneas de la efectividad del entrenamiento. La evaluación de la eficacia diferencial de un Programa para subgrupos homogéneos puede ayudar a los investigadores a obtener una nueva visión del éxito de la intervención más precisa. Existen investigaciones que han investigado el efecto diferenciador de las intervenciones sobre los participantes, pero estas experiencias son escasas. Pocos estudios han investigado los efectos diferenciales de Programas de entrenamiento de ARA por tanto, la presente investigación se considera un aporte. Sin embargo, es necesario seguir investigando sobre cómo se pueden adaptar las intervenciones a las necesidades de diferentes grupos de estudiantes para obtener resultados de entrenamiento óptimos y aumentar la efectividad de los programas de ARA (Lourenco y Ferreira, 2019).

\section{Limitaciones y orientaciones para futuros estudios en esta área de investigación}

El desafío para la futura investigación es diseñar un entrenamiento docente breve, con prácticas de instrucción específicas para docentes universitarios que apoye la promoción de ARA de sus estudiantes. Este entrenamiento para la práctica educativa del docente puede integrar orientaciones sobre el fomento de variables de ARA en el aula en diversos contextos de aprendizaje, incluyendo aquellos que involucran uso de plataformas virtuales (Valencia y Caicedo, 2017). Además, sería importante desarrollar una investigación que implique la comparación de la evaluación del impacto de programas para el desarrollo de competencias de autorregulación en formato intracurricular y en formato extracurricular. De esta forma se podría evidenciar que un mismo programa con las características de intracurricular (implicación del docente de asignatura disciplinar en el desarrollo de competencias de autorregulación, y el desarrollo de actividades específicas en el contexto de la asignatura), probablemente es más eficaz que en su formato extracurricular (Merchan y Hernández, 2018).

Otra orientación para la futura investigación es considerar aspectos referidos a la evaluación del impacto de programas de entrenamiento. Es importante utilizar diferentes técnicas para medir las variables de autorregulación, y que las mediciones se realicen en diferentes momentos respecto de la exposición al programa que busca mejorar esta competencia, no sólo medidas pre y pos test. Con el primer aspecto se puede verificar la discrepancia entre lo que el estudiante conoce y lo que usa realmente una determinada estrategia, con el segundo aspecto se podría evaluar la estabilidad del tratamiento en el tiempo de las mejoras logradas con el Programa (Díaz et al., 2017).

\section{CONCLUSIONES}

Basado en los resultados de este estudio y en la discusión presentada que compara resultados de otras investigaciones, se establecen las siguientes cinco conclusiones:

1) el Programa Intracurricular Para la Disposición al Estudio resultó una intervención efectiva para potenciar estrategias y creencias de autorregulaión en estudiantes de primer año de ingeniería;

2) la modalidad intracurricular del programa de intervención mostró un impacto significativo en la promoción de competencias de autorregulación;

3) existe un efecto diferencial del efecto del Programa considerando la pertenencia de los estudiantes a una determinada carrera; 
4) los docentes tiene un rol clave en la promoción intracurricular de promoción del aprendizaje autorregulado en sus estudiantes; $y$

5) se considera este estudio un aporte a la Educación Superior ya que entrega directrices acerca de cómo mejorar en contexto de aula variables asociadas a la primera fase de autorregulación del aprendizaje, a través de una intervención basada en evidencia científica, que ha mostrado resultados válidos y que apoya resultados encontrados a nivel internacional poniendo a disposición de investigadores un programa de entrenamiento para estudiantes que cursan primer año en la universidad.

\title{
AGRADECIMIENTOS
}

Trabajo realizado el marco del proyecto de investigación "Impacto de un programa de entrenamiento en estrategias y creencias de disposición al estudio" para optar al grado de Doctor en Psicología de la Universidad de Concepción, Chile. Cuenta con financiamiento de Becas Doctorado Nacional CONICYT Folio 21161224; y con apoyo de los proyectos FONDECYT № 1161502 y FONDEF ID17/10393.

\section{NOTACIÓN}

\author{
Símbolos \\ G.C $=\quad$ Grupo control en tabla 3 y 4 \\ G.E $=\quad$ Grupo experimental en tabla 3 y 4 \\ ${ }^{* *}=\quad p<0.05$ en tabla 3 y 4 \\ * $\quad p<0.1$ en tabla 3 y 4 \\ Dif. $\quad$ Diferencia en tabla 3 y 4 \\ $X^{2}(1)=\quad$ Resultado de la prueba de razón de verosimilitud entre el modelo con y sin efecto de interacción grupo \\ tiempo en tabla 3 \\ $\eta_{\mathrm{G}}=\quad$ Eta generalizado en tabla 3 y 4 \\ $X^{2}(2)=\quad$ Resultado de la prueba de razón de verosimilitud entre el modelo con y sin efecto de interacción grupo \\ tiempo en tabla 4
}

\section{REFERENCIAS}

Ali, M., Self-regulated Learning Pedagogy for Teaching Applied Engineering and Technology Class, Journal of Technology, Management, and Applied Engineering, 35(1), 1-11 (2019).

Barr, S., y H., Askell-Williams, Changes in Teachers' Epistemic Cognition About Self-Regulated Learning as They Engaged in a Researcher-Facilitated Professional Learning Community, doi: 10.1080/1359866X.2019.1599098, Asia-Pacific Journal of Teacher Education, 1(2), 1-26 (2019).

Bloom, M., Self-Regulated Learning: Goal Setting and Self-Monitoring, The Language Teacher, 37(4), 46-51 (2013).

Burke, A., Student Retention Models in Higher Education: A Literature Review, College and University, 94(2), 12-21 (2019).

Concannon, J., Serota, S. y otros dos autores, How interests, Self-efficacy, and Self-Regulation Impacted six Undergraduate Pre-engineering Students' Persistence, doi: 10.1080/03043797.2017.1422695, European Journal of Engineering Education, 44(4), 1-20 (2019).

de la Fuente, J., Mañas, I. y otros tres autores, Differential Effect of Level of Self-Regulation and Mindfulness Training on Coping Strategies Used by University Students, doi. 10.3390/ijerph15102230, International Journal of Environmental Research and Public Health, 15(10), 1-20 (2018).

Díaz, A., Pérez, M. y otros dos autores, Impacto de un Entrenamiento en Aprendizaje Autorregulado en Estudiantes Universitarios, Perfiles Educativos, ISSN: 0185-2698, 39(157), 87-104, (2017).

Dowd, J., Thompson, R. y otros siete autores, Student Learning Dispositions: Multidimensional Profiles Highlight Important Differences Among Undergraduate STEM Honors Thesis Writers, doi: 10.1187/cbe.18-07-0141, CBE Life Sciences Education, 18(2), 18-28 (2019).

Duchatelet, D., y Donche, V., Fostering Self-efficacy and Self-regulation in Higher Education: A Matter of Autonomy Support or Academic Motivation?, doi: 10.1080/07294360.2019.1581143, Higher Education Research and Development, 38(4), 733-747 (2019).

García-Ros, R., y Pérez-González, F., Validez Predictiva e Incremental de las Habilidades de Autorregulación sobre el Éxito Académico en la Universidad, Revista de Psicodidactica, ISSN: 1136-1034, 16(2), 231-250, (2011).

García-Ros, R., y Pérez-González, F., Spanish Version of the Time Management Behavior Questionnaire for University Students, doi: 10.5209/rev_SJOP.2012.v15.n3.39432, The Spanish Journal of Psychology, 15(3), 1485-1494 (2012). 
Graffigna, A., Hidalgo. L., y otros cuatro autores, Tutorial Practice as a Strategy of Retention at the School of Engineering, 10.1016/j.sbspro.2014.01.598, Procedia - Social and Behavioral Sciences, 116(1), 2489-2493 (2014).

Hernández, A., y Camargo, A., Autorregulación del Aprendizaje en la Educación Superior en lberoamérica: Una Revisión Sistemática, doi: 10.1016/j.rlp.2017.01.001, Revista Latinoamericana de Psicología, 1(49), 146-160 (2017).

Inglés, C., Rodríguez-Marín, J. y González-Pienda, J., Adaptación de la Sydney Attribution Scale en Población Universitaria Española, Psicothema, ISSN: 0214-9915, 20(1), 166-173, (2008).

King, B., Hamilton, L. y Johnson, C., First-year Students' Self-regulation Process Through Self-report at a Minority Serving Institution (MSI). Journal of Education, doi: 10.9734/jesbs/2019/v30i330130, Society and Behavioural Science, 30(3), 1-8 (2019).

Lattuada, M., Deserción y Retención en las Unidades Académicas de Educación Superior. Una Aproximación a las Causas, Instrumentos y Estrategias que Contribuyen a Conocer y Morigerar su Impacto, Debate Universitario, ISSN: 23142138, 5(10), 100-113, (2017).

Lawanto, O., Febrian, A. y otros dos autores, Self-regulation Strategies in an Engineering Design Project, doi: 0.5539/ies.v12n5p133, International Education Studies, 12(5), 133-152 (2019).

Loeffler, S., Bohner, A. y otros tres autores, Investigating and fostering self-regulated learning in higher education using interactive ambulatory assessment, doi: 10.1016/j.lindif.2019.03.006, Learning and Individual Differences, 71(2), 43-57 (2019).

Lourenco, D., y Ferreira, A., Self-regulated Learning and Training Effectiveness, doi: 10.1111/ijtd.12149, International Journal of Training and Development, 23(2), 117-134 (2019).

Merchan, N., y Hernández, N., Rol Profesoral y Estrategias Promotoras de Autorregulación del Aprendizaje en Educación Superior, Espacios, ISSN: 0798-1015, 39(52), 1-12, (2018).

Olejnik, S., y Algina, J., Generalized Eta and Omega Squared Statistics: Measures of Effect Size for Some Common Research Designs, doi: 10.1037/1082-989X.8.4.434, Psychological Methods, 8(4), 434-447 (2003).

Pérez-Villalobos, M. V., Cobo-Rendón, R. C. y otros dos autores, Revisión Sistemática de la Habilidad de Autocontrol del Estudiante y su Rendimiento Académico en la Vida Universitaria, doi: 10.4067/S0718-50062018000300049, Formación Universitaria, 11(3), 49-62. (2018).

Pinxten, M., Van Soom, C. y otros tres autores, At-risk at the Gate: Prediction of Study Success of First-year Science and Engineering Students in an Open-admission University in Flanders-any Incremental Validity of Study Strategies?, doi: 10.1007/s10212-017-0361-x, European Journal of Psychology of Education, 34(1), 45-66 (2019).

Sáez, F., Bustos, C. y Díaz, A., Autoeficacia Cuestionario de Autorregulación de Estudio Readiness, doi: 10.15689/ap.2017.1701.10.13348, Avaliação Psicológica, 17(1), 92-100 (2018a).

Sáez, F., Bustos, C. y otros cuatro autores, Disposición al Estudio, Autoeficacia y Atribuciones Causales en Estudiantes Universitarios Chilenos, doi: 10.20511/pyr2018.v6n1.179, Propósitos y Representaciones, 6(1), 199-245 (2018b).

Sáez, F., Díaz, A. y otros dos autores, Revisión Sistemática Sobre Competencias de Autorregulación del Aprendizaje en Estudiantes Universitarios y Programas Intracurriculares para su Promoción, doi: 10.4067/s0718-50062018000600083, Formación Universitaria, 11(6), 83-98 (2018c).

Valencia, M., y Caicedo, A., Diseño de Tareas Apoyadas en TIC para Promover Aprendizaje Autorregulado, doi: 10.11144/Javerianacali.PPSI15-2.dtat, Pensamiento Psicológico, 15(2), 15-28 (2017).

Wang, J., Liu, K. y Ma, G., The Role of Social-Academic Goals in Chinese Students' Self-regulated Learning, doi: 10.1007/s10212-018-0404-y, European Journal of Psychology of Education, 34(3), 579-600 (2019).

Weiner, B., The Development of an Attribution-based Theory of Motivation: A History of Ideas, doi: 10.1080/00461520903433596, Educational Psychologist, 45(1), 28-36 (2010).

Zeidner, M., Self-regulated Learning: Current Fissures, Challenges, and Directions for Future Research, doi: 10.1080/13598139.2019.1584034, High Ability Studies, 1(2), 1-22 (2019). 